\title{
Editorial
}

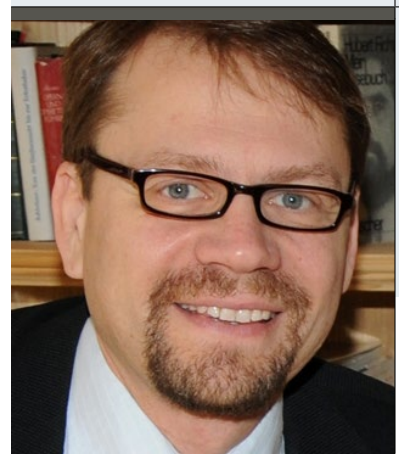

"Das Wichtigste: Selbst aktiv daran denken! Menschen mit

einer fortgeschrittenen Demenz thematisieren ihre Schmerzen

nicht von sich aus ..."

Prof. Dr. med. Michael Hüll

Chefarzt der Klinik für Alterspsychiatrie und Psychotherapie,

Zentrum für Psychiatrie Emmendingen

\section{Schmerzen bei Demenz}

E sibt keinen Grund anzunehmen, dass Menschen mit Demenz weniger häufig als gleichaltrige an Schmerzen leiden. Komorbiditäten wie Dekubiti oder Kontrakturen machen es eher noch wahrscheinlich, dass die Schmerzbelastung höher liegen könnte. Ab einem schweren Demenzstadium machen die eingeschränkte Kommunikation sowie nicht wahrgenommene Schonhaltungen das Erkennen dieser Schmerzen äußerst schwierig. Der CME-Beitrag von Albert Lukas macht auf das Dilemma aufmerksam, dass wir auch mit für Menschen mit Demenz entwickelten Schmerzskalen und Instrumenten dieses Problem nicht gänzlich lösen können. Dabei ist ein systematischer Ansatz, zum Beispiel mit der „Verbalen Rating-Skala“, sicherlich unsystematischen Einschätzungsversuchen überlegen. Am wichtigsten ist aber, selber aktiv daran zu denken, da Menschen mit einer fortgeschrittenen Demenz Schmerzen nicht von sich aus thematisieren können.

\section{Probatorischer Einsatz zeigt Effekte, ist aber nicht unproblematisch}

Die von Christine Kovach in den USA entwickelte "Serial Trial Intervention" geht dabei fast noch einen Schritt weiter: Nach dieser soll bei Verhaltensstörungen bei Menschen mit Demenz auch ohne erkennbaren Schmerzfokus probatorisch ein Versuch mit einem Schmerzmittel erfolgen. Die komplexen Vorgehensweisen der Serial Trial Intervention wurden nicht nur in den USA, sondern auch in Deutschland und den Niederlanden untersucht. Insgesamt zeigten sich in zumeist clusterrandomisierten Studien kleine positive Effekte, wobei der Beitrag des Schmerzmitteleinsatzes im Rahmen der komplexen Intervention schwierig abzuschätzen bleibt.
Andererseits sind Schmerzmittel im Alter keine unproblematischen Substanzen. Die eingeschränkte Verträglichkeit von NSAID, seien es Blutungen (insbesondere in Verbindung mit NOAK) oder Nierenprobleme, führen oft zu einer raschen Empfehlung von Opioiden. Opiode, die aus genannten Gründen bei Schmerzen bei Älteren zu Recht einen frühen Einsatz finden, sind nicht nur Schmerzmittel, sondern auch psychotrope Substanzen mit multiplen Wirkungen wie Sedierung und serotonergen Effekten. Dies kann bis zu serotonergen Syndromen etwa bei der Kombination von Fentanyl mit einem SSRI gehen. Verändern Schmerzmittel das Verhalten, ist nicht immer auf Schmerzen als Auslöser des Verhaltens zu schließen. Auch Gewöhnungseffekte mit Wirkverlust und Absetzeffekte sind zu befürchten.

Die derzeit heftig diskutierte unkritische ärztliche Verordnung von Opioiden in den USA und England ist sicherlich nicht durch den Einsatz bei älteren Menschen mit Demenz bedingt. Die dabei aber wieder deutlich gewordene Schwierigkeit, wieder einen Ausstieg aus einem Therapiebegin zu finden, sollte uns bewusst sein.

Aufgrund der aktuell eher bestehenden Unterversorgung mit Schmerzmitteln sind zurzeit alle Versuche, diese zu beheben zu begrüßen. Überschießende Ansätze nach der Devise "Gebt dem Opi Opium, dann ist Opi vielleicht stumm" sollten jedoch nicht aufkommen.

$\mathrm{Ihr}$

Michael Hüll 